\title{
"Sandwich technique" enables preservation of hearing and antivertiginous effect in cholesteatomatous labyrinthine fistula
}

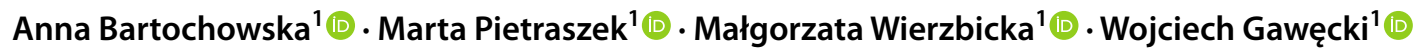

Received: 12 April 2021 / Accepted: 9 June 2021 / Published online: 18 June 2021

(c) The Author(s) 2021

\begin{abstract}
Purpose The aim of the study was to assess hearing, surgical and clinical results of the treatment in patients with cholesteatomatous labyrinthine fistula (LF) focusing on the different techniques and materials used in the management.

Methods Study group included 465 patients. Cases with LFs discovered or confirmed during surgical procedure were thoroughly analyzed.

Results LFs were noted in $11.4 \%$ of all cases. Thirty-eight patients, with all follow-up data available, were included into the further analysis. Most LFs were located in the lateral semicircular canal (87\%). LFs were assessed as small in 2 cases, as medium in 24 patients while 12 were described as large. Based on Dornhoffer and Milewski classification, $50 \%$ of LFs were classified as IIa, 24\% as IIb, 6 LFs were very deep (type III), while 4-superficial (type I). The size and type of LF did not influence postsurgical complaints ( $p=0.1070, p=0.3187$, respectively). Vertigo was less frequent in LFs treated by "sandwich technique", especially those with opened endosteum. In 30 (79\%) patients, hearing improved or did not change after surgery. Hearing outcomes were significantly better in the ears operated by means of CWU technique $(p=0.0339)$, in LFs with intact membranous labyrinth $(p=0.0139)$ and when "sandwich technique" was performed $(p=0.0159)$. Postsurgical bone conduction thresholds levels were significantly better in LFs covered by "sandwich method" $(p=0.0440)$.

Conclusion "Sandwich technique" (temporal fascia-bone pate-temporal fascia) enables preservation of hearing as well as antivertiginous effect in patients with cholesteatomatous labyrinthine fistula.
\end{abstract}

Keywords Labyrinthine fistula $\cdot$ Cholesteatoma $\cdot$ Intratemporal complication $\cdot$ Vertigo

\section{Introduction}

Labyrinthine fistula (LF) is the most common intratemporal complication of chronic otitis media with cholesteatoma. According to the literature, it concerns $4-12 \%$ of the ears operated due to this pathology $[1,2]$. It mostly affects lateral semicircular canal $(87 \%)$ and rarely promontorium (8\%), superior (6\%) and posterior part of the labyrinth (2\%) [2]. Preoperative detection of LF based on symptoms and clinical examination is often not specific and not sensitive enough [3]. High-resolution computed tomography (HRCT) significantly improves these indicators allowing proper diagnosis in $85-100 \%$ cases [3-5]. However, management of

Anna Bartochowska

annabartochowska@gmail.com

1 Department of Otolaryngology, Head and Neck

Surgery, Poznan University of Medical Sciences, 49

Przybyszewskiego Street, 60-355 Poznan, Poland this pathology still remains controversial. Despite years of disputes on the best method of treatment providing both closure of the fistula and preservation of hearing, otosurgeons constantly debate whether "to leave the matrix over fistula or to remove it completely" $[1,2]$, which approach-canal wall up (CWU) or canal wall down (CWD) — should be used $[2,3,5]$, if intraoperative steroid administration improves the results $[6,7]$. As far as we know, only one group of researchers [7] analyzed the impact of the technique of the fistula closure on the final outcomes.

The aim of the study was to assess hearing, surgical and clinical results of the treatment in patients with cholesteatomatous labyrinthine fistula focusing on the different techniques and materials used in their management. 


\section{Materials and methods}

Our study group included all patients operated on in our department due to cholesteatoma in the years 01.2015-03.2020 (465 altogether). We selected those with labyrinthine fistula discovered or confirmed during surgical procedure. These cases were thoroughly analyzed (patients hospitalized in 2015-2017 retrospectively while those treated in 2018-2020 mostly prospectively).

The following variables were collected: age, gender, comorbidities, signs and symptoms, otosurgical history, pre- and postoperative air and bone conduction thresholds (at $0.5,1,2$ and $3 \mathrm{kHz}$ ), fistula characteristics (location, size and type), HRCT scans of the temporal bone, surgical technique (canal wall up versus canal wall down approach; method of LF closure), and perioperative steroid use.

Fistula size was described according to the Sanna et al. [8] who divided them into small $(0.5-1 \mathrm{~mm})$, medium (1-2 $\mathrm{mm})$ and large (>2 mm). Fistula-type assessment based on Dornhoffer and Milewski classification are [6]: I-fistula with erosion of the bony labyrinth with an intact endosteum; II-fistula with opened endosteum but intact membranous labyrinth (IIa-with undisturbed perilymphatic space; IIb where perilymphatic spaces were disturbed either by active accidental suctioning before recognition of the fistula or by ingrowth of the cholesteatoma); and III-fistula with open perilymphatic space with concomitant involvement or destruction of the underlying membranous labyrinth.

Cholesteatoma location, extent and advancement were described basing on the European Academy of Otology and Neurotology/Japanese Otological Society joint consensus staging system [9].

HRCT scans of the temporal bones were assessed preoperatively both by radiologists and otosurgeons.

All surgeries were performed by two experienced otosurgeons. The choice of surgical approach was independent of the presence of the fistula. It was mostly determined by cholesteatoma characteristics, patient's general state and past ear history. CWD approach was usually chosen in cases with extensive, rapidly recurrent pathology, in difficult anatomical conditions as well as in patients with contraindications to perform another surgery in general anesthesia. In all cases with LF, removal of the cholesteatoma matrix from the fistula was performed after complete dissection of the pathology from the middle ear spaces. Since 2016 in most LFs mesna was additionally used to facilitate LF cleansing (a small amount (1-3 ml) of $20 \%$ saline solution of mesna was administered topically for ca. 2 min). We did not drill nor suction at the site of LF. We also did not perform labyrinthectomy or obliteration of LF. In all cases, cholesteatoma matrix was completely removed from the fistula in one-stage procedure. LF was immediately covered by autogenous material collected and prepared before the dissection of the matrix from the region of LF. The method of the fistula closure was mostly dependent on LF stage. In most type I LFs, bone pate was used, while in types II and III, bone pate and temporal fascia. In 2018, we started to perform "sandwich technique" in most cases of LFs with opened endosteum (Figs. 1 and $2 \mathrm{a}-\mathrm{c})$. A small layer patch of fascia was placed over the open fistula, then bone pate and again temporal fascia to stabilize two previous sheets. Intraoperative steroids were introduced into the treatment scheme of LF in our department in 2016. Sixteen milligrams of dexamethasone were given intravenously by anesthesiologists at the time of cholesteatoma removal. In all patients from the study group, steroids were administered intravenously after surgery at the ward ( $8 \mathrm{mg}$ of dexamethasone twice a day for at least 3 days).

All patients were regularly followed up. In patients operated with CWU technique with no clinical signs of recurrent disease, non-echoplanar diffusion-weighted imaging magnetic resonance (MRI DWI NON EPI) was done 1 and 2 years after the surgery. In patients with recidivistic disease, reoperations were performed.

For statistical purposes, in the assessment of hearing outcomes, air and bone conduction thresholds from the audiograms performed at least 10 months after the surgery were considered.

Statistical analysis was conducted to determine factors influencing treatment outcomes. We focused on the method of fistula closure. Because of small sample size and more than $20 \%$ of cells with expected frequencies less than 5 , we used Fisher Exact test for comparison of variables. All calculations were performed at the level of alpha $=0.05$ using R software.

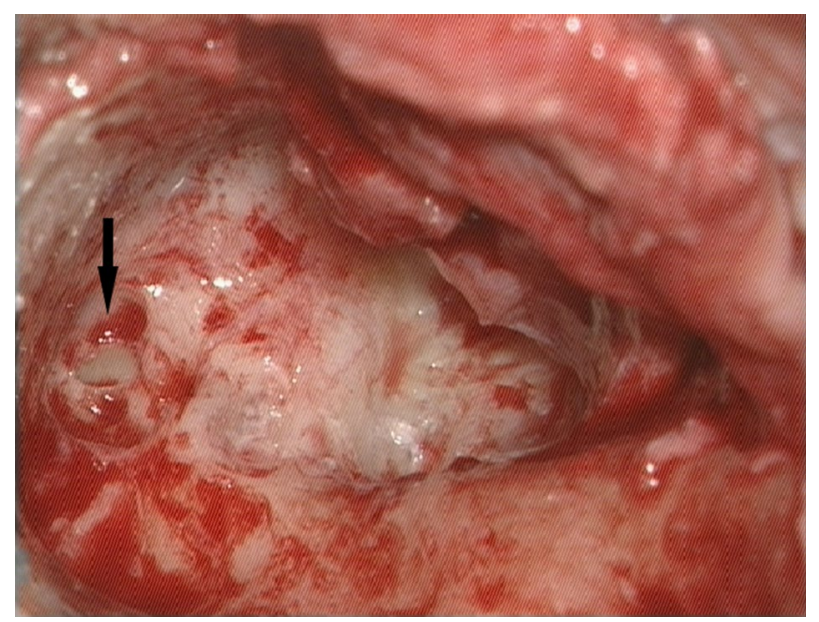

Fig. 1 LF with opened endosteum before the closure 

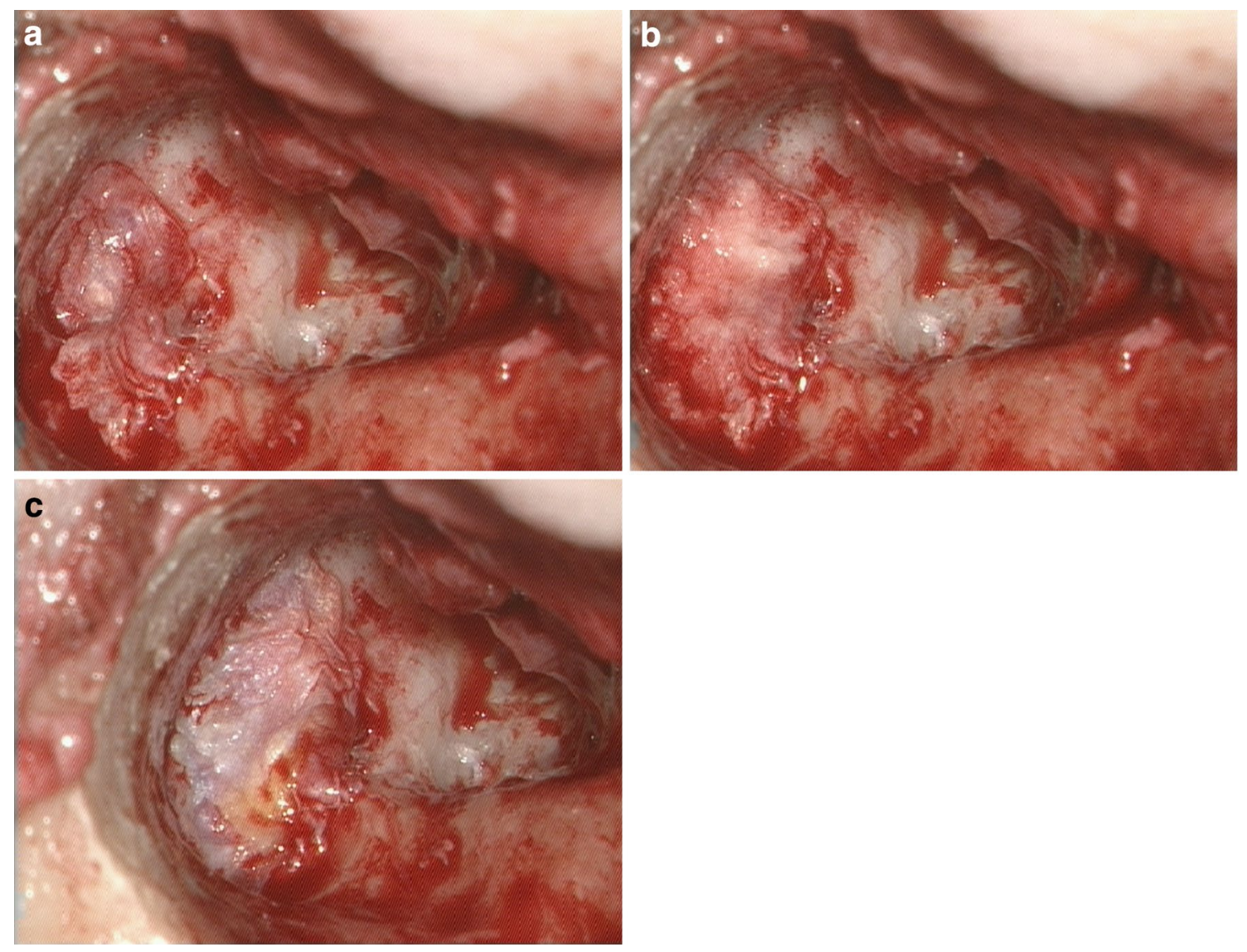

Fig. 2 "Sandwich technique" as a method of LFs treatment, a first layer: temporal fascia, b second layer: bone pate, $\mathbf{c}$ third layer: temporal fascia)

The study was approved by our institutional ethics committee.

\section{Results}

LF was noted in 53 out of 465 analyzed patients which constituted $11.4 \%$ of all cases operated on due to the cholesteatoma in our department. Thirty-eight cases, with all follow-up data available, were included into the further analysis.

\section{Epidemiological data/comorbidities}

There were 20 women and 18 men. Mean age of patients at the time of surgery was 47 years (range 11-76 years). Twelve patients suffered from hypertension, three were treated for diabetes, while one for hypothyroidism, one for asthma and one for chronic kidney disease (Table 1).

\section{Preoperative clinical data}

Most patients reported symptoms typical for cholesteatoma: ear discharge (68\%) and hearing loss (89\%). Vertigo and dizziness were present in 18 cases-in 12 patients, the course of the disease was gradual while in 6-with rapid onset. In only 12 cases, a positive fistula sign was found. In 7 patients, nystagmus was examined, in 2 beating towards the healthy ear.

Primary surgery was performed in 20 cases. Eighteen patients have been operated before (10 in other departments), out of which 9 more than once.

In 10 patients, conductive hearing loss was detected, in 25-mixed hypoacusis while 3 ears were deaf prior to surgery. The preoperative mean bone conduction level was $29 \mathrm{~dB}$ while mean air threshold was at the level of $57 \mathrm{~dB}$.

All preoperative clinical data are presented in Table 1.

Based on HRCT the presence of fistula was described by radiologists in 26 cases and detected preoperatively by otosurgeons in 30 imagings. 
Table 1 Clinical characteristics of patients with labyrinthine fistula (LF)

\begin{tabular}{|c|c|}
\hline Mean age (years) & $\begin{array}{l}47.32 \pm 16.40 \mathrm{SD} \\
\text { Number of patients }(\%)\end{array}$ \\
\hline \multicolumn{2}{|l|}{ Sex } \\
\hline Female & $20(52.63)$ \\
\hline Male & $18(47.37)$ \\
\hline \multicolumn{2}{|l|}{ Comorbidities } \\
\hline Hypertension & $12(31.58)$ \\
\hline Diabetes & $3(7.89)$ \\
\hline Hypothyroidism & $1(2.63)$ \\
\hline Asthma & $1(2.63)$ \\
\hline Chronic kidney disease & $1(2.63)$ \\
\hline \multicolumn{2}{|l|}{ Signs and symptoms } \\
\hline Ear discharge & $26(68.42)$ \\
\hline Hearing loss & $34(89.47)$ \\
\hline Vertigo & $18(47.37)$ \\
\hline \multicolumn{2}{|l|}{ Clinical examination } \\
\hline Positive fistula sign & $12(32.58)$ \\
\hline Nystagmus & $7(18.42)$ \\
\hline \multicolumn{2}{|l|}{ Otosurgical history } \\
\hline Primary surgery & $20(52.63)$ \\
\hline Second procedure & $9(23.68)$ \\
\hline History of 2 or more procedures & $9(23.68)$ \\
\hline \multicolumn{2}{|l|}{ Cholesteatoma location in the middle ear (STAM) } \\
\hline \multicolumn{2}{|l|}{ Difficult-to-reach recesses $(\mathrm{S})$ : } \\
\hline Protympanum (S1) & $11(28.95)$ \\
\hline Sinus tympani (S2) & $14(36.84)$ \\
\hline Tympanic cavity $(\mathrm{T})$ & $20(52.63)$ \\
\hline $\operatorname{Attic}(\mathrm{A})$ & $26(68.42)$ \\
\hline Mastoid process (M) & $36(94.74)$ \\
\hline \multicolumn{2}{|l|}{ Cholesteatoma advancement } \\
\hline I. One location & - \\
\hline II. Two or more locations & - \\
\hline III. With extracranial or intratemporal complications & $37(97.37)$ \\
\hline IV. With intracranial complications & $1(2.63)$ \\
\hline \multicolumn{2}{|l|}{ Hearing results } \\
\hline Conductive hypoacusis & $10(26.32)$ \\
\hline Mixed hypoacusis & $25(65.79)$ \\
\hline Deafness prior to surgery & $3(7.89)$ \\
\hline
\end{tabular}

\section{Intraoperative findings}

Most LFs were located in the lateral semicircular canal (LSC) (87\%), in 2 patients-in superior semicircular canal (SSC), while in 3-LFs were multiple (in LSC and SSC). LFs were assessed as small in 2 cases, as medium-in 24 patients while 12 were described as large. Based on Dornhoffer and Milewski classification, $50 \%$ of LFs were classified as IIa, 24\% as IIb, 6 LFs were very deep (type III), while 4-superficial (type I) (Table 2).

In 8 cases, LF was detected intraoperatively (patients had no symptoms neither HRCT showed the presence of LF).
In $53 \%$ of cases, cholesteatoma was located in the tympanic cavity (T), in $68 \%$ in attic (A) and in $95 \%$ in mastoid process (M). In 14 patients, sinus tympani (S2) was involved, in 11 protympanum (S1). Most cholesteatomas (97\%) were evaluated as stage III (Table 1).

There were found no statistically significant differences between cholesteatoma location and advancement and fistula size ( $p=0.9999, p=0.9999$, respectively) and type $(p=0.1833, p=0.5000$, respectively $)$. 
Table 2 LF characteristics

\begin{tabular}{lc}
\hline & $\begin{array}{c}\text { Number of } \\
\text { patients (\%) }\end{array}$ \\
\hline LF location & $33(86.84)$ \\
LSC & $2(5.26)$ \\
SCC & $3(7.89)$ \\
Multiple LFs & \\
LF size & $2(5.26)$ \\
Small & $24(63.16)$ \\
Medium & $12(31.58)$ \\
Large & \\
LF type & $4(10.52)$ \\
I & $19(50.00)$ \\
IIa & $9(23.68)$ \\
IIb & $6(15.79)$ \\
III & fistula, $L S C$ \\
\hline$L F \quad$ labyrinthine \\
lateral semicircular canal, SCC \\
superior semicircular canal
\end{tabular}

\section{Surgical management}

CWU technique was chosen in 19 cases. CWD was also performed in 19 patients (out of whom 10 have been operated by CWD method previously). In 15 cases, mesna was additionally used. In all the ears, there were no signs of residual cholesteatoma in neither middle ear spaces nor fistula region at the end of surgery. In 12 cases, bone pate was used to close LF, in 9-bone pate and temporal fascia, while in 17 "sandwich technique" was performed. Intraoperative steroids were used in 20 cases. In all the patients, intravenous steroids were prescribed in postoperative time and given for 3-7 days (mean 5 days).

\section{Postoperative outcomes}

Postoperatively, ten patients (26\%) presented vertigo (which lasted for 3-30 days) — six of them reported dizziness before the surgery as well. In all these cases, LFs were medium or large, in 3 cases-type IIb, in 3-type III, while in 4-IIa. The size and type of LF did not influence postsurgical complaints ( $p=0.1070, p=0.3187$, respectively). Although it is not confirmed in statistical analysis $(p=0.1604)$, we noted that postoperative vertigo was less frequent in LFs treated by "sandwich technique", especially those with opened endosteum.

In $30(79 \%)$ patients, hearing (defined as a summary mean air and bone conduction gain) improved or did not change after surgery. In 6 cases, air and bone conduction threshold levels lowered (mean hearing loss: $25 \mathrm{~dB}$ for air and $10 \mathrm{~dB}$ for bone conduction). Two patients presented postoperative deafness. In all LFs treated with "sandwich technique", hearing was preserved or improved. Hearing outcomes were significantly better in the ears operated by means of CWU technique ( $p=0.0339)$, in LFs with intact membranous labyrinth $(p=0.0139)$ and when "sandwich technique" was performed ( $p=0.0159)$. Postsurgical bone conduction threshold levels (defining inner ear function) were significantly better in LFs covered by "sandwich method" $(p=0.0440)$ (Table 3).

The mean follow-up time in the study group was 23 months (range 10-48 months). In 3 patients, residual disease was detected in MRI DWI NON EPI, in other 3 cases recurrence was diagnosed in otoendoscopy. In all recidivistic ears, cholesteatoma was confirmed in reoperations. There were no cases of recurrent LFs in the study group.

\section{Discussion}

The management of LF has been a subject of debate for years. In the literature, there is still the lack of consensus in the treatment schemes. In fact, optimal guidelines do not exist.

The first point of controversy still regards the technique (CWU or CWD) that should be performed in case of cholesteatomatous LF. Years ago, most otosurgeons recommended radical or modified radical mastoidectomy as the only methods allowing sufficient visualization of LFs and their proper management $[10,11]$. Nowadays, most authors underline that the decision on preservation of posterior canal wall should not be determined by the presence of LF but should depend on many factors including hearing status of the operated and contralateral ear, location and extension of the cholesteatoma, coexistence of other complications, patient's age and general condition $[3,5,12]$. However, when we analyze results presented in the latest literature, it turns out that CWD seems to be preferred technique in LF cases (Table 4) [1, 5, 13-16]. While in some departments CWD is still the method of choice [15], detailed analysis shows that most surgeons choose the approach that primarily enables them radical removal of the cholesteatoma. Although in our study group, 50\% of patients were treated by CWD technique, we underlined that the choice of the method was dependent on cholesteatoma related factors and was not determined by the presence of LF itself. What important, almost $50 \%$ of our patients have been operated before ( $26 \%$ by CWD) which could probably pave the way for LF development (too strenuous drilling in the region of LF during the previous procedures?) or indicate the aggressive pattern of cholesteatoma growth with increased bone destruction processes. In our opinion, if possible, CWU approach should be used as the first-line treatment in all LF cases. Moreover, extreme caution regarding thorough HRCT analysis (also by otosurgeons regarding the fact that, if not directed by information in the referral form, radiologist can 


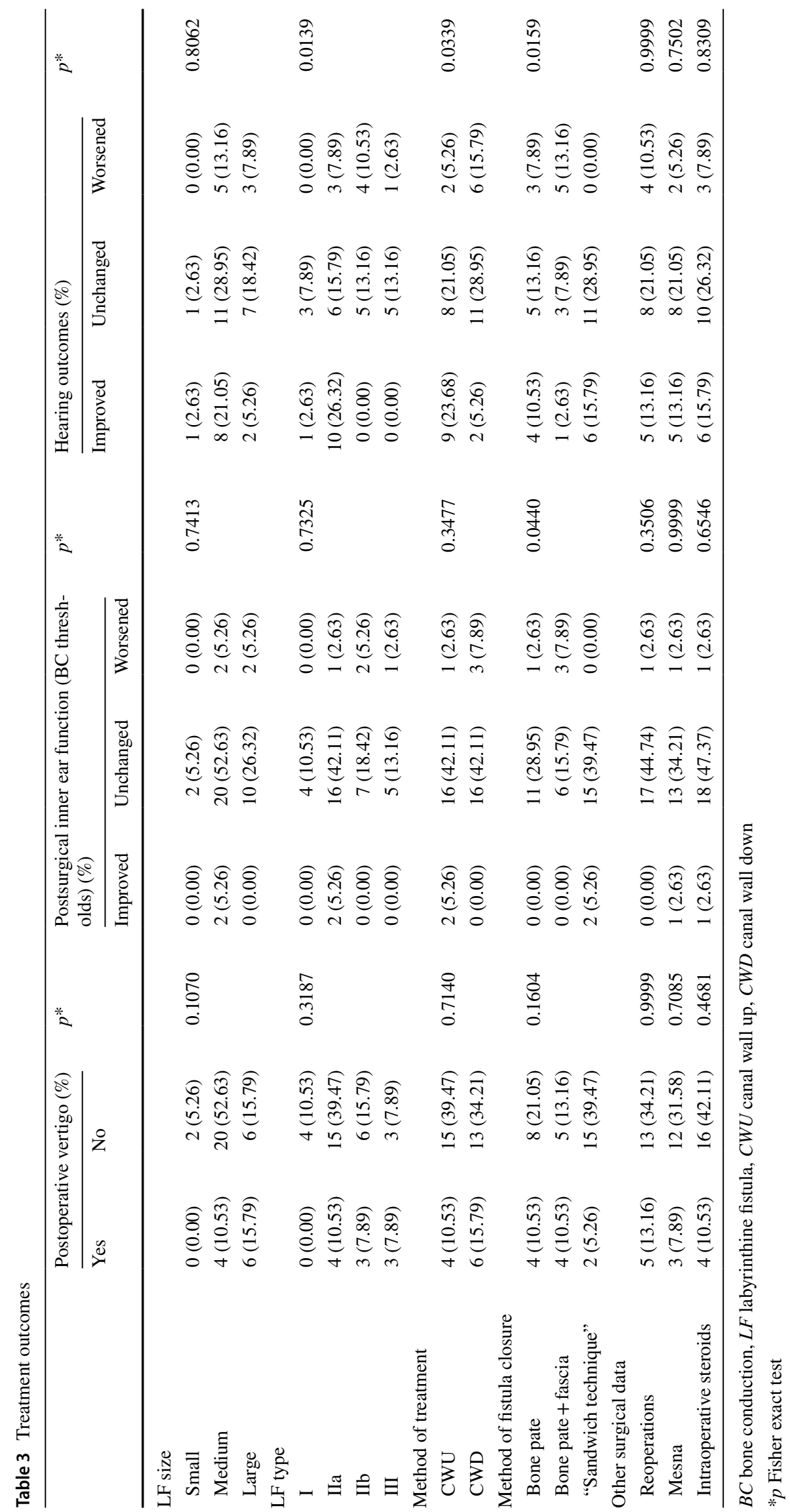




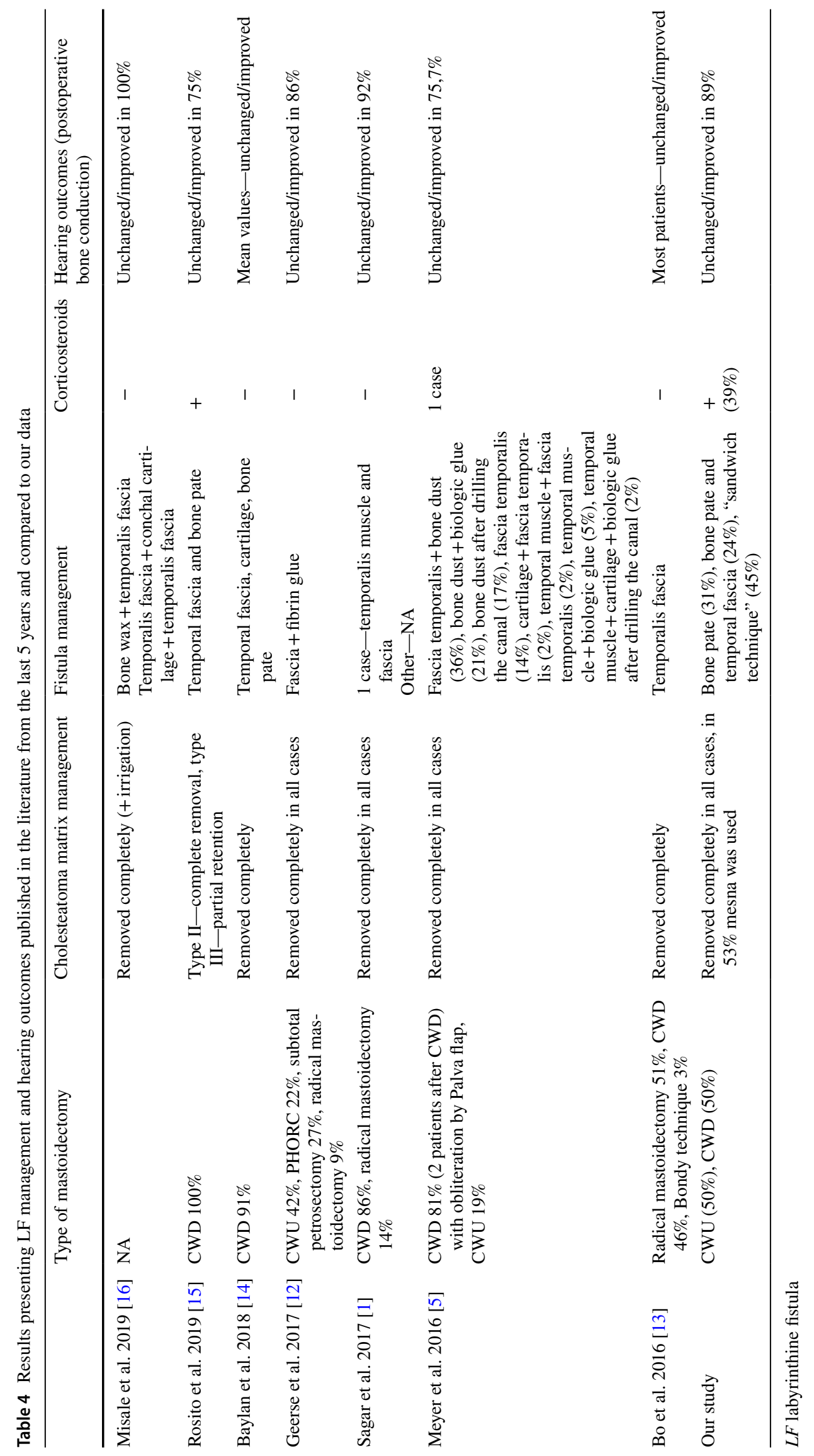


miss describing the presence of LF-_—we had such a situation in several cases) and delicate surgical dissection of cholesteatoma in the region of semicircular canals should be maintained in all reoperated ears.

The second point of dispute concerns the issue of the removal of the cholesteatoma from the LF [1, 7]. Fortunately, the idea of leaving the matrix over LF was preferred years ago $[8,17]$. Many studies show that the risk of hearing loss by labyrinthine damage during the dissection of cholesteatoma matrix from the LF area is equal when compared to the risk of its damage by collagenases present in residual epithelium [18]. Moreover, preservation of the cholesteatoma in the region of LF may lead to delayed complications like suppurative labyrinthitis or meningitis [10]. Therefore, precise dissection of the matrix from the fistula seems to be a mainstay of the proper treatment. In our study, radical removal of the cholesteatoma was performed in all cases. We paid attention to delicate preparing of the epithelium from LF, in most cases preceded by the use of mesna, without suctioning and drilling in its region. We think that such a protocol should be recommended in all LFs.

In the literature, we found only one article analyzing the impact of the material used in LF closure on the final outcomes. Gocea et al. [7] did not find the differences between conchal cartilage, bone dust, periosteum and the mixture of them on the clinical results. Other otosurgeons, except for the tissues listed above, preferred temporal fascia, temporal muscle, bone wax or biologic glue in different configurations. We are advocates of "sandwich technique" introduced in our department in 2018. Previously we tended to use bone pate or bone pate covered by temporal fascia. We noticed that patients in whom LFs with opened endosteum were sealed with bone pate presented more intense and longer dizziness than those in whom LFs were covered by temporal fascia first. It was probably caused by small bone particles that entered semicircular canals and behaved like free-floating otoliths. On the other hand, fascia alone, especially when used in CWD approach, had a tendency to relocate. It also seems to be insufficient material to close LF-patients after CWD with such a reconstruction very often experience vertigo when exposed to the cold wind while people treated by CWU method feel dizzy in situations of increased pressure in the middle ear. It encouraged us to close LFs with three layers-small patch of temporal fascia over the open fistula, then bone pate and again temporal fascia to stabilize two previous sheets. "Sandwich method" did not only reduce postoperative vertigo in our patients but also improved hearing outcomes. It was probably due to better closure of LF. Jang et al. [19] achieved good antivertiginous results by obliterating CWD mastoid cavities. They believed that obliterative mass can form a barrier that protects labyrinth from induced pressure changes. Our three sandwich layers probably acted the same.
In many articles, the protective role of intraoperative steroids on hearing is being raised $[6,7,19]$. They have antioxidative and antiapoptotic activity, antiproliferative effect on cholesteatoma regrowth in the LF site as well as the ability to stabilize intralabyrinthine membranes $[6,7,20]$. The method was introduced to LF management by Dornhoffer and Milewski [6] — they used intraoperatively $500 \mathrm{mg}$ injection of methylprednisolone at the time of LF cleansing and observed beneficial impact of such a procedure on treatment outcomes in LFs with open labyrinth. Considering steroid induced side effects, we decided to use smaller doses $-16 \mathrm{mg}$ of dexamethasone which equals $85 \mathrm{mg}$ of methylprednisolone. Although some authors [12] suggest that steroids do not improve hearing results, we think that they can have positive effect on that key treatment outcome.

Among methods facilitating removal of the matrix from the fistula laser [21], special techniques, e.g., underwater ear surgery [22] and mesna [23] are described. We do not have experience with the first mentioned method, but we appreciate significant role of mesna in atraumatic LF clearance. We recommend it in the management of cholesteatomatous LF as well.

\section{Conclusions}

"Sandwich technique" (temporal fascia-bone pate-temporal fascia) enables preservation of hearing as well as antivertiginous effect in patients with cholesteatomatous labyrinthine fistula. Radical gentle removal of the matrix from LF, without suctioning and drilling in its region, facilitated by topical mesna use and intraoperative intravenous injection of steroids, followed by immediate covering of LF is a safe LF management protocol. The choice of surgical approach should not be determined by the presence of LF but should depend on hearing status of the operated and contralateral ear, location and extension of the cholesteatoma, coexistence of other complications, patient's age and general condition. Extreme caution regarding thorough HRCT analysis as well as delicate surgical dissection of cholesteatoma in the region of semicircular canals should be maintained in all reoperated ears.

Author contributions All the authors whose names appear on the submission, (1) made substantial contributions to the conception or design of the work; or the acquisition, analysis, or interpretation of data; or the creation of new software used in the work; (2) drafted the work or revised it critically for important intellectual content; (3) approved the version to be published; and (4) agree to be accountable for all aspects of the work in ensuring that questions related to the accuracy or integrity of any part of the work are appropriately investigated and resolved. Conceptualization: BA; methodology: BA, PM, and GW; formal analysis and investigation: BAand PM; writing—original draft preparation: BA; writing - review and editing: PM, WM, and GW; supervision: WM and GW. 
Funding No funding was received for conducting this study.

Data availability Yes.

Code availability MS Excel.

\section{Declarations}

Conflict of interest The authors have no relevant financial or non-financial interests to disclose.

Ethics approval Approval was obtained from the ethics committee of Poznan University of Medical Sciences. The procedures used in this study adhere to the tenets of the Declaration of Helsinki.

Consent to participate Informed consent was obtained from all individual participants included in the study.

Consent for publication Patients signed informed consent regarding publishing their data and photographs.

Open Access This article is licensed under a Creative Commons Attribution 4.0 International License, which permits use, sharing, adaptation, distribution and reproduction in any medium or format, as long as you give appropriate credit to the original author(s) and the source, provide a link to the Creative Commons licence, and indicate if changes were made. The images or other third party material in this article are included in the article's Creative Commons licence, unless indicated otherwise in a credit line to the material. If material is not included in the article's Creative Commons licence and your intended use is not permitted by statutory regulation or exceeds the permitted use, you will need to obtain permission directly from the copyright holder. To view a copy of this licence, visit http://creativecommons.org/licenses/by/4.0/.

\section{References}

1. Sagar P, Devaraja K, Kumar R, Bolu S, Sharma SC (2017) Cholesteatoma induced labyrinthine fistula: is aggressiveness in removing disease justified? Indian J Otolaryngol Head Neck Surg 69:204-209. https://doi.org/10.1007/s12070-017-1072-y

2. Copeland BJ, Buchman CA (2003) Management of labyrinthine fistulae in chronic ear surgery. Am J Otolaryngol 24:51-60. https://doi.org/10.1053/ajot.2003.10

3. Stephenson MF, Saliba I (2011) Prognostic indicators of hearing after complete resection of cholesteatoma causing a labyrinthine fistula. Eur Arch Otorhinolaryngol 268:1705-1711. https://doi. org/10.1007/s00405-011-1545-7

4. Ikeda R, Kobayashi T, Kawase T, Oshima T, Sato T (2012) Risk factors for deterioration of bone conduction hearing in cases of labyrinthine fistula caused by middle ear cholesteatoma. Ann Otol Rhinol Laryngol 121:162-167. https://doi.org/10.1177/00034 8941212100304

5. Meyer A, Bouchetemblé P, Costentin B, Dehesdin D, Lerosey Y, Marie JP (2016) Lateral semicircular canal fistula in cholesteatoma: diagnosis and management. Eur Arch Otorhinolaryngol 273:2055-2063. https://doi.org/10.1007/s00405-015-3775-6

6. Dornhoffer JL, Milewski C (1995) Management of the open labyrinth. Otolaryngol Head Neck Surg 112:410-414. https://doi.org/ 10.1016/s0194-5998(95)70275-x

7. Gocea A, Martinez-Vidal B, Panuschka C, Epprecht P, Caballero M, Bernal-Sprekelsen M (2012) Preserving bone conduction in patients with labyrinthine fistula. Eur Arch Otorhinolaryngol 269:1085-1090. https://doi.org/10.1007/s00405-011-1757-x

8. Sanna M, Zini C, Gamoletti R, Taibah AK, Russo A, Scandellari $\mathrm{R}$ (1988) Closed versus open technique in the management of labyrinthine fistula. Am J Otol 9:470-475

9. Yung M, Tono T, Olszewska E, Yamamoto Y, Sudhoff H, Sakagami M, Mulder J, Kojima H, İncesulu A, Trabalzini F, Özgirgin N (2017) EAONO/JOS joint consensus statements on the definitions, classification and staging of middle ear cholesteatoma. J Int Adv Otol 13:1-8. https://doi.org/10.5152/iao.2017.3363

10. Palva T, Kärjä J, Palva A (1971) Opening of the labyrinth during chronic ear surgery. Arch Otolaryngol 93:75-78

11. Freeman P (1978) Fistula of the lateral semicircular canal. Clin Otolaryngol Allied Sci 3(3):315-321

12. Geerse S, de Wolf MJF, Ebbens FA, van Spronsen E (2017) Management of labyrinthine fistula: hearing preservation versus prevention of residual disease. Eur Arch Otorhinolaryngol 274:3605-3612. https://doi.org/10.1007/s00405-017-4697-2

13. Bo Y, Yang Y, Xiaodong C, Xi W, Keyong T, Yu Z, Ye W, Kun L, Zheng Y, Yang C, Jianhua Q (2016) A retrospective study on post-operative hearing of middle ear cholesteatoma patients with labyrinthine fistula. Acta Otolaryngol 136:8-11. https://doi.org/ 10.3109/00016489.2015.1087650

14. Baylan MY, Yılmaz Ü, Akkuş Z, Topçu İ (2018) Assessment of bone conduction thresholds after surgical treatment in patients with labyrinthine fistula. Turk Arch Otorhinolaryngol 56:89-94. https://doi.org/10.5152/tao.2018.3238

15. Rosito LPS, Canali I, Teixeira A, Silva MN, Selaimen F, Costa SSD (2019) Cholesteatoma labyrinthine fistula: prevalence and impact. Braz J Otorhinolaryngol 85:222-227. https://doi.org/10. 1016/j.bjorl.2018.01.005

16. Misale P, Lepcha A, Chandrasekharan R, Manusrut M (2019) Labyrinthine fistulae in squamosal type of chronic otitis media: therapeutic outcome. Iran J Otorhinolaryngol 31:167-172

17. Sheehy JL, Brackmann DE (1979) Cholesteatoma surgery: management of the labyrinthine fistula-a report of 97 cases. Laryngoscope 89:78-87

18. Lim J, Gangal A, Gluth MB (2017) Surgery for cholesteatomatous labyrinthine fistula. Ann Otol Rhinol Laryngol 126:205-215. https://doi.org/10.1177/0003489416683193

19. Jang CH, Jo SY, Cho YB (2013) Matrix removal of labyrinthine fistulae by non-suction technique with intraoperative dexamethasone injection. Acta Otolaryngol 133:910-915. https://doi.org/10. 3109/00016489.2013.782105

20. Mori T, Fujimura K, Yoshida M, Suzuki H (2004) Effects of glucocorticoid receptor antagonist on CAPs threshold shift due to short-term sound exposure in guinea pigs. Auris Nasus Larynx 31:395-399. https://doi.org/10.1016/j.anl.2004.09.008

21. Basu S, Hamilton J (2019) Treatment using diffuse laser energy of cochlear and vestibular fistulas caused by cholesteatoma. J Laryngol Otol 133:102-105. https://doi.org/10.1017/S0022215119000173

22. Yamauchi D, Yamazaki M, Ohta J, Kadowaki S, Nomura K, Hidaka H, Oshima T, Kawase T, Katori Y (2014) Closure technique for labyrinthine fistula by "underwater" endoscopic ear surgery. Laryngoscope 124:2616-2618. https://doi.org/10.1002/lary.24785

23. Vincenti V, Magnan J, Saccardi MS, Zini C (2014) Chemically assisted dissection by means of mesna in cholesteatoma surgery. Otol Neurotol 35:1819-1824. https://doi.org/10.1097/MAO.00000 00000000514

Publisher's Note Springer Nature remains neutral with regard to jurisdictional claims in published maps and institutional affiliations. 